\title{
Adding TQ-BOT into a Third-party Learning Management System
}

\section{Añadiendo TQ-BOT en un Learning Management System proporcionado de Terceros}

\begin{abstract}
Intelligent Tutoring Systems are computer programs that aim at providing personalized instruction to students. In recent years, artificial intelligence conversational robots, usually known as chatterbots, have become very popular in the Internet. In this paper we show how chatterbots can be integrated in e-Learning Systems. To perform such an integration the Service Oriented Architecture paradigm is adopted and e-learning standardization initiatives are considered. A middleware is provided to enable the integration and reuse of chatterbots by e-Learning systems enabling a tight control of their operation. Such middleware takes to account several issues such as authorising users, creating instances, transferring data to and from the chatterbot, assigning permissions to users, and subscribing to events. Our approach is applied to the specific case of TQ Bot, which is use to track and supervise the student progress and to provide answers orienting the student to the more Keywords: Chatterbot. Agent Models. E-learning. Keywords: Chatterbot. Agent Model Resumen: Los Sistemas de Tutorización Inteligentes son
programas informáticos que tienen como objetivo proporcionar enseñanza personalizada a los estudiantes. En los últimos años, los robots de inteligencia artificial de conversación, por lo general conocidos como chatterbots, se han vuelto muy populares en el Internet. En este trabajo se muestra cómo se puede integrar chatterbots en sistemas de e-Learning. Para llevar a cabo esta integración se adopta el paradigma de arquitectura orientada a servicios y algunas iniciativas de estandarización. En el artículo se describe un middleware para permitir la integración y la reutilización de chatterbots por los sistemas de e-learning que permite una un estricto control de su funcionamiento. El middleware es necesario para desarrollar varios aspectos, tales como autorizar a los usuarios, la creación de instancias, la transferencia de datos hacia y desde el chatterbot, la asignación de permisos a los usuarios, y suscribirse a los eventos. Nuestro enfoque se aplica al caso concreto de TQBot, que es utilizado para el seguimiento y supervisión de progreso de los estudiantes y para proporcionar respuestas que orienten al alumno en el curso.

Palabras-chave: Chatterbots. Modelos de Agentes. Elearning. Middleware. Arquitectura Orientada a Servicios.
\end{abstract}

CAEIRO-RODRIGUEZ Manuel; MIKIC-FONTE, Fernando Ariel; FONTENLA-GONZALEZ, Jorge, PEREZ-RODRIGUEZ, Roberto; BURGUILLO-RIAL, Juan Carlos; LLAMAS NISTAL, Martin. Adding TQ-BOT into a Third-party teoria \& Mrática, Porto Alegre, v, 14, n 1, 4 43-59: jan./jun. 2011.

\section{Manuel Caeiro-Rodríguez Universidade de Vigo}

Fernando Ariel Mikic-Fonte Universidade de Vigo

Jorge Fontenla-González Universidade de Vigo

Roberto Pérez-Rodríguez Universidade de Vigo

Juan Carlos Burguillo-Rial Universidade de Vigo

Martín Llamas-Nistal

Universidade de Vigo

\section{Introduction}

$\mathrm{T}$ he adoption of new technologies in education is continually increasing. The Internet's functionality and capability is being applied to support an increasing number of courses at different levels (from $\mathrm{K}-12$, to higher education, to lifelong learning), in a broad range of disciplines, and in different contexts (e.g. distance learning, blended learning or traditional in-class education). In some cases, e-Learning systems are used to supplement existing learning activities, such as lecturing, fact-finding and experimentation. In other cases, the 
systems support brand new activities, such as organizing personalized learning materials and providing instant assessment through online tests. In any case, technology is becoming a main tool to support educational processes at all levels.

Artificial Intelligence (AI) is usually considered as a key technology domain in the development and adoption of e-learning systems. Since the 1980 's many research projects have been devoted to the development of Intelligent Tutoring Systems (ITSs) (CORBETT et al., 1997), intelligent agents, and more specifically the use of conversational agents, usually called chatterbots, which allow the communication with users in natural language.

A.L.I.C.E. (Artificial Linguistic Internet Computer Entity) (ALICE A. I. Foundation, 1995) has been one of the most groundbreaking projects in the field of AI during the last years. A.L.I.C.E. is the project that produced the AIML (Artificial Intelligence Markup Language) through which is posible to develop software chatterbots (NEVES et al., 2002). A.L.I.C.E. has won the "Loebner Prize in Artificial Intelligence Contest" (based on the Turing test) several times. An A.L.I.C.E.-like chatterbot can be used as a tutor in an e-learning system to provide tutoring and evaluating support. In this paper we use an A.L.I.C.E. based chatterbot named TQ-Bot, which is used to track and supervise the progress of the students, and to provide answers orienting them to the more appropriate course contents.

A main issue in the use of chatterbots is their integration in e-learning systems. Chatterbots are usually developed ad-hoc and with no interoperability support. Today we can find many bots in the literature (BURGUILLO, 2008; ALICE A. I. Foundation, 1995; PIETRO; FRONTERA, 2004; LEONHARDT et al., 2003), but it is very difficult to use them in contexts different from the one they were conceived for. This can be seen as a reusability problem that should be solved.

In this paper we show a solution based on the Service Oriented Architecture (SOA) programming paradigm that enables the integration of chatterbots into e-learning systems. This work extends some standardization initiatives in the e-learning domain for the integration of third-party tools (ALCOM et al., 2006). Our solution comprises a middleware, interfaces and protocols to achieve a hard integration of third-party tools and e-learning systems involving transparency and privacy requirements key for final users. As a result, it is provided an infrastructure that can be used to support the integration of chatterbots in e-learning systems. In this paper we show how a specific chatterbot (TQ-Bot) is integrated into a SOA-based LMS using this infrastructure.

The rest of the paper is organized as follows. Next section introduces the context of the paper in the e-learning domain. Section 3 provides a general view of a common e-learning system, identifying key parts and components. Following this introduction the integration middleware is described in Section 4, and Section 5 includes the adoption of this infrastructure to support the integration of a chatterbot in an e-learning system. The paper ends up with some conclusions.

\section{Background}

Nowadays, the most common e-learning systems are Learning Management Systems (LMSs) (WEAVER, 2002; ZEMSKY et al., 2009). LMSs are deployed as holistic platforms intended to manage all the issues involved in distance learning. These issues comprise authoring, assessing and delivering tools to provide specific functionalities (e.g. profile management tools, productivity tools, communication tools). In their first attempts, LMSs were essentially repositories with lots of documents but very basic functionality. However, these platforms evolved into rich environments where students can communicate, collaborate, access to multimedia files, participate in virtual worlds, subscribe to podcasts, writing wikis, playing games, etc. The Edu-Tools (WCET, 2009) review analyses 39 different LMSs.

In spite of the advantages of LMSs, there exist some important drawbacks that should not be overlooked. The lack of a tutor figure to pay specific attention to a individual 
students is one of these drawbacks. Here is where a chatterbot can play an important role. A chatterbot can be dedicated to tutoring students, taking advantage of $\mathrm{AI}$ techniques, and to offer a kindly interface to the users. This bot can help students at any time of the day, any day of the week. It does not get bored or loses its patience due to the students' attitude, and it can attract and keep students' attention because it supposes a technological innovation. Even to some degree, a chatterbot can make the student feel more comfortable than just surfing through the learning resources and tasks.

Attending to the development model, current LMSs can be grouped into two main categories (DAGGER et al., 2007). The first category is about open source initiatives (such as Moodle, .LRN, Sakai, dotLRN, ATutor, Whiteboard), which are built over extensible frameworks that let implementers adjust and modify the systems to match their specific needs. The other category involves proprietary solutions (such as WebCT/Blackboard, Gradepoint, Desire2Learn, Learn.com). These systems support extensions by providing software developers with "hooks" to tie third-party software into the LMS. Nevertheless, there is not any solution that can be applied in a general way.

The need for extensibility solutions in elearning systems has led many organizations to develop and publish several standards and recommendations. Some standards regard the definition of layered and decoupled architectures (DAGGER et al., 2007). Examples of these are the E-Learning Framework (ELF) (JISC and DEST, 2004), the IMS Abstract Framework (IMS-AF) (Smythe, 2003) and the Open Knowledge Initiative (OKI) (OKI, 2001). Among the targets of these specifications we can find the modularization of functionality in elearning systems by the identification of well-defined core components, interfaces and APIs. These elements are defined to support the interoperability with the other elements via Web Services, and grouped according to their functionality (DAGGER et al., 2007). However, the practical adoption of these works is very limited, and therefore they are regarded just as theoretical frameworks. Other kind of specifications (IMS General Web Services (SCHROEDER et al., 2005), IMS Tool Interoperability (ALCOM et al., 2006) and IMS Common Cartridge (UNJHEM et al., 2008)) are related to the extension of the functionalities of current elearning systems by means of their interconnection with third-party components during runtime, using broadly-accepted Web technologies and paradigms such as SOAP,WSDL, UDDI (WALSH, 2002), Ajax and Comet (CRANE;MCCARTHY, 2008), Saas (TRUMBA CORPORATION, 2004), IaaS (HAMAMO, 2009), and Cloud Computing (JONES, 2009). Despite their heterogeneity, these solutions present well-known advantages in terms of interactivity and scalability.

\section{Architecture of an e-Learning system}

In this section we describe the software architecture used to integrate e-learning systems and third-party tools. We use a general description of an e-learning system, since the scope of the presented solution does not restrict to any particular platform. Our exposition begins with the educational scenario concept, which agglutinates pedagogical and computational requirements into a computerunderstandable building block for online courses.

\subsection{The Educational Scenario concept}

The educational scenario is the fundamental unit for constructing complex courses. The most relevant elements for defining an educational scenario are participants, which are enrolled into scenarios; goals, which declare learning objectives; environments, which aggregate learning resources and tools (in which bots are included); and temporal deadlines, which indicate the temporal limit for fulfilling goals. Therefore, an educational scenario encapsulates a fully functional unit of learning. 

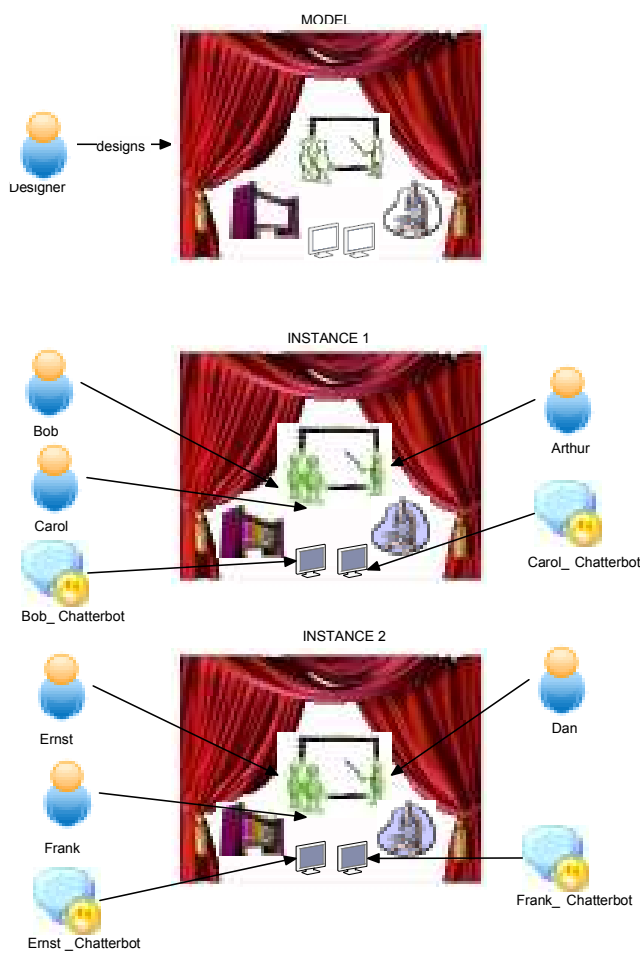

Figure 1 - Design time and runtime of an educational scenario

The life-cycle of educational scenarios can be divided into the following stages: design time, instantiation time and runtime. The concept of scenario is, therefore, twofold: it can be whether the model created during design time, or a concrete instance, with concrete participants enrolled into, and with certain temporal constraints as well. These concepts are illustrated in Figure 1. In design time, the author creates the model of the scenario using an authoring tool. In the example, a scenario with human participants and chatterbots is depicted, as well as a lab environment with some tools: a microscope and some books on inorganic chemistry. In instantiation time, a new instance of the educational scenario is created from the model in order to handle a particular case. In the example of the figure, three participants are grouped and enrolled into the first educational scenario instance: Arthur, having a teacher's role; Bob and Carol, having a learner's role; and Bob Chatterbot and Carol Chatterbot, having a consultant role. In the same way, Dan, Ernst, Frank, Dan Chatterbot and Ernst
Chatterbot are grouped and enrolled into the second educational scenario instance. The creation of a new scenario instance entails creating instances of its containing elements: a new environment instance as well as instances for tools and chatterbots into the environment. Finally, in runtime, participants access to environment instances and make use of tools and chatterbot instances. Notice that every group of participants use its own scenario instance.

In the following subsection we detail a general architecture to support the life-cycle of educational scenarios, from design time to runtime.

\subsection{General architecture}

We present the structure of a general elearning system as composed of three layers: Presentation Layer, Business Logic Layer, and Database Layer. In Figure 2 this structure is depicted.

- The Presentation Layer displays educational scenarios, making use of the functionality provided by the Business Logic Layer. Presentation components are designed following a decomposition according to its three main functionalities:

- The Authoring component is employed to design educational scenarios.

- The Monitoring component is employed to monitor educational scenarios. An authorized user can check the state of an educational scenario, as well as the progression of participants in an educational scenario.

- The Delivering component displays participants' working space.

- The Business Logic Layer is the core component of the e-learning system. It manages information related to educational scenarios, participants, tools, chatterbots, and the rest of elements, by using the persistence capabilities of the Database Layer. The Business Logic Layer is integrated into the e-learning system through a welldefined interface, therefore guaranteeing connectivity 
requirements. The two main functionalities provided by the Business Logic Layer are models management and instances management.

- The Models Manager is in charge of managing educational scenario models. It provides an authoring interface for creating the models.

- The Instances Manager deals with managing educational scenario instances.

An event such as finishing a learning activity may trigger several events inside the Business Logic Layer, such as the assignment of the assessment of that learning activity to a qualified teacher. Communication between the Presentation Layer and the Instances Manager may be passive information retrieval as well as the communication of events generated by participants.

- Finally, the Database Layer maintains two separate schemas: one for educational scenario models, and another one for educational scenario instances.

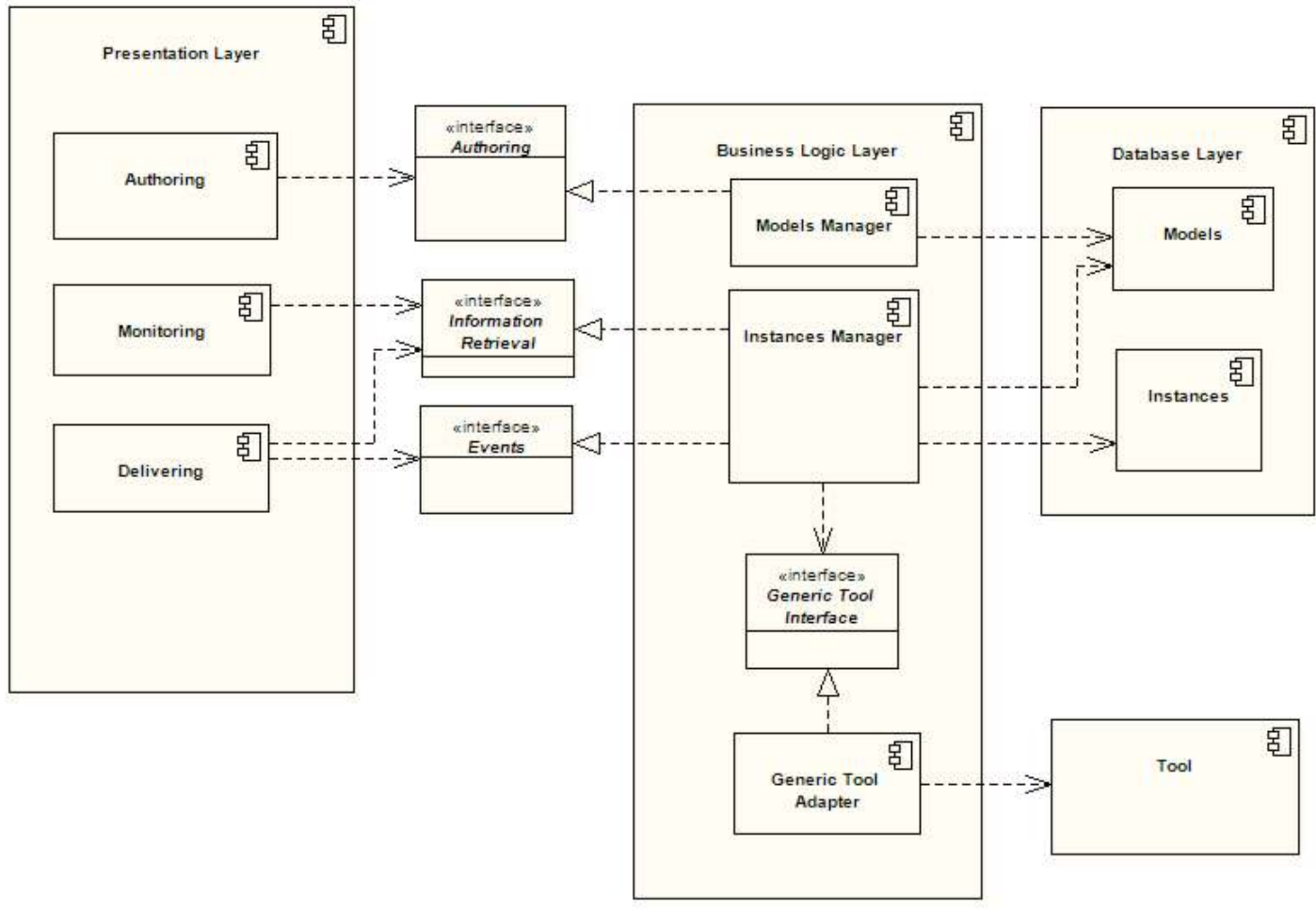

Figure 2 - General architecture of an e-learning System

\subsection{Our LMS}

Following the guidelines presented in the latter subsection, we have developed an LMS. The presentation layer of our LMS is inspired in Moodle (MOODLE, 2002), programmed in PHP. The presentation layer counts with views for authoring, monitoring, and delivering.

The Business Logic Layer is based on the PoEML (CAEIRO, 2007), which is an Educational Modelling Language and, as such, it allows to describe scenarios, groups of participants, tools, resources, and the rest of elements in educational scenarios. This layer enables the definition and execution of learnflows (PEREZ et al., 2009) involving participants, learning goals, temporal constraints, etc. This layer is implemented as a Java Web Application running on Tomcat (APACHE, 2000).

The Business Logic Layer is integrated in the overall system through a well-defined interface that is based on Web Services. This approach provides the maximum level of interoperability in web-based scenarios. In order to make Web Services accessible to presentation modules, we use the 
functionalities provided by a SOAP engine, Axis (APACHE, 2004). The functionalities that the Business Logic Layer provides are published in a WSDL file. The service methods serve for passive information retrieval, communication of events, and adhoc changes in instances. The JavaToWSDL tool provides for automatic WSDL generation from Java code. The WSDL file is automatically generated from the Java class containing the declaration of Web Service methods as a Java interface definition.

In the Presentation Layer we use the NuSOAP (AYALA; NICHOL, 2009) library, which facilitates the consumption of Web Service methods. After retrieving the WSDL file containing the definition of Web Service methods, the Presentation Layer is able to declare a client and request service methods from the Business Logic Layer.

The Database Layer is implemented on Oracle (ORACLE, 2009). We have chosen Oracle because of its good out-of-the-box scalability support, which is an important concern in big e-learning deployments, as those of universities supporting distance learning courses.

\section{Seamless integration of chatterbots in e-Learning Systems}

Given the previous architecture of a generic e-learning system, our objective in this section is to describe an extension mechanism in order to complement the basic features of the system with the aid of third-party tools, in this case chatterbots. We consider that the integration of new functionalities must be as tight as possible, and must be carried out with minimum changes in the legacy systems. In the following sections we give some definitions concerning the level of integration of a thirdparty tool in an e-learning system, and then we provide a close look to the architecture we have developed for integrating of chatterbots.

\subsection{Soft and hard integration}

At this point we consider two opposite alternatives for integrating thirdparty tools in e-learning systems, which are also considered in (KYNG, 1997):

- Soft integration of third-party tools. The e-learning system functionality can be extended through a hyperlink to an (external) third-party component. When the user clicks on it, the graphical user interface of the tool is displayed. From this point, users are operating a tool that the e-learning system cannot control by any means. Therefore, a new functionality is included but it does not work in coordination with the core system, resulting in a very "soft" integration.

- Hard integration of third-party tools. It includes soft integration, but providing the e-learning system with a more comprehensible control over the integrated tools. We describe in the next paragraphs our proposal for such a comprehensible control.

Hard integration allows the e-learning system not only to link the application, but also to supervise and alter the workflow of the tool as required, in order to adapt it to the concrete requirements and limitations of the course and its users.

As discussed in (CAEIRO, 2007), the control of the operation of a learning tool (a chatterbot in this paper) to achieve hard integration in e-learning systems involves the following issues:

1. Creating a chatterbot instance for each user. For example, in an "Chemical" subject a chatterbot instance can be created for helping a learner in the course.

2. Transferring from the e-learning system to the chatterbot all those data that the user may need in order to carry out his/her tasks. In the previous example the student can obtain additional content asking the chatterbot. Previously, the chatterbot received such content from the elearning system.

3. Establishing some access permissions over these data and the chatterbot functionality. In our example, the student may be assigned a configuration permission to change 
certain features of the bot, for example its name or background image.

4. Subscribing to events result of the work with the chatterbot. For example, the e-learning system may be interested in knowing when the student access to some specific contents provided by the bot.

5. Authorising the user to access the chatterbot instance. In our example, the student may not have access credentials at the chatterbot, in whose case the e-learning system has to grant him/her access as guest user.
6. Activating an action in the chatterbot according to the information provided by the events triggered. For example, the LMS activate a message in the chatterbot to inform the learner that 5 minutes remain to finish the task.

Figure 3 summarizes the differences between hard and soft integration in terms of the six aspects mentioned above. Nonetheless, it should be kept clear that soft and hard integration represent extreme alternatives, and it is possible to conceive intermediate solutions.

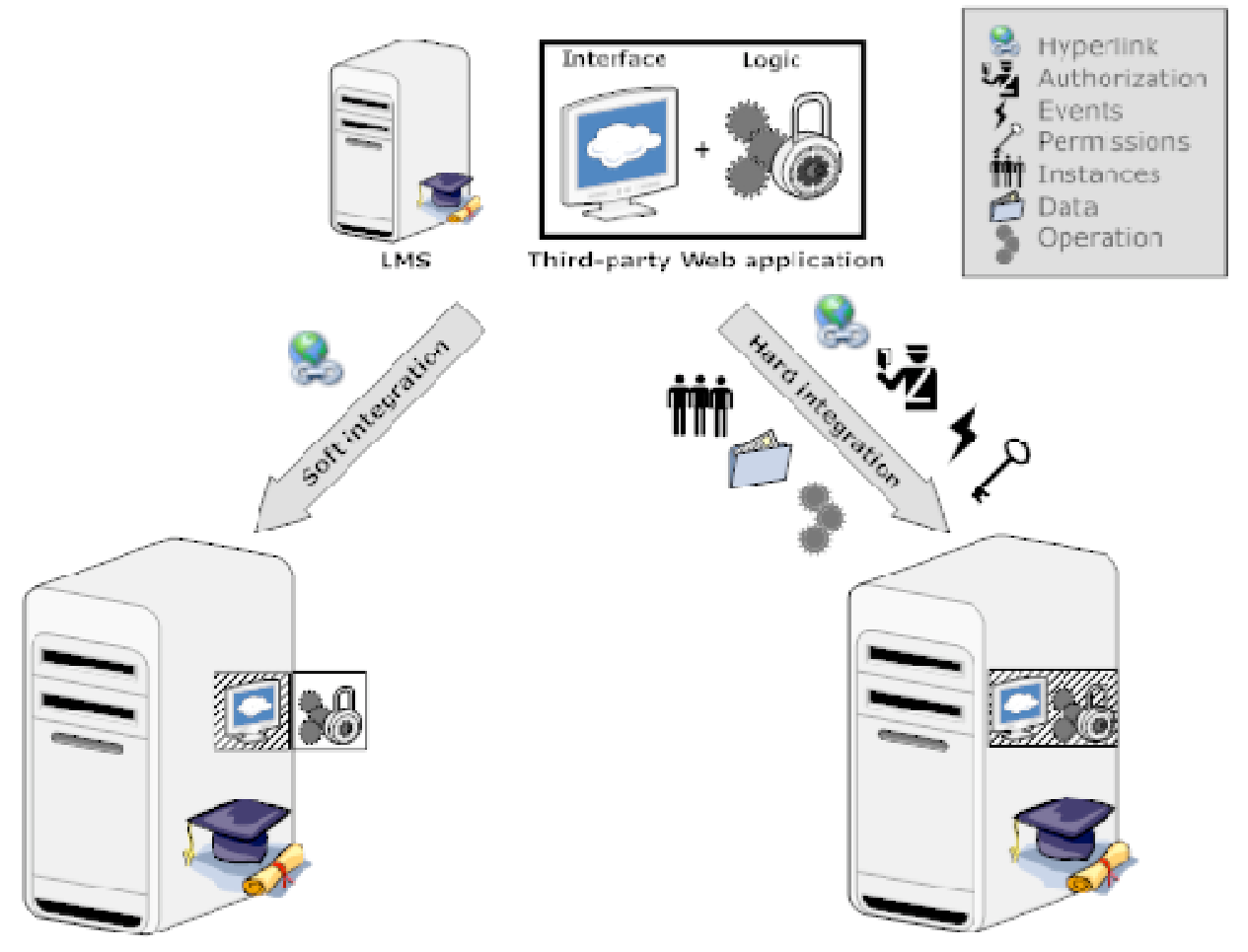

Figure 3 - Comparison between hard integration and soft integration in e-learning systems

\subsection{The generic tool adapter}

The Generic Tool Adapter has been posed as a software component to extend the functionalities of an e-learning system by enabling the integration of third-party tools in a hard way. In the context of this research work a chatterbot is considered as a special kind of third-party tool. This adapter has been developed at our research group to allow e-learning systems to import, control and manage external tools that complement the functionalities of the LMS. The aspects covered by this adapter involve:

1. Authorization granting. A single sign-on mechanism, named Reverse OAuth (FONTENLA et al., 2009), included as part of the Generic Tool Adapter, has been developed in order to authorize users (e.g. learners and teachers) to access the tool without requiring additional sing-ins. This is especially interesting when users have already authenticated after the e-learning systems and, from their point of view, 
additional authentications after the tool should not be necessary.

2. Instances management. The Generic Tool Adapter includes resources devoted to control the instances of the tool. We understand by instance of a tool a working environment along with a graphical user interface, associated to several files to manipulate, and a set of users allowed to access it. Several methods are included to control the creation and deletion of concrete tool instances, and to add and remove users to tool instances.

3. Data transfer. A mechanism to exchange data between the LMS and the tool, either single data values or full backups of user data. This functionality allows the e-learning system, for example, to submit configuration files to a chatterbot and to get a log of conversations in the chatterbot.

4. Permissions assignment. A functionality is included in order to set Access permissions to specific users over concrete parts of the tool. This functionality provides an straightforward mechanism to differentiate the different roles of teachers and students (e.g. students may be allowed to communicate with a chatterbot and teachers, additionally, may have permissions to change its configuration).

5. Event subscription. This feature allows the e-learning system to subscribe to particular events triggered by the tool in response to specific actions carried out by its users. This feature is specially useful in elearning environments, where the external system must be "in touch" with what happens inside the tool in order to track, evaluate and help students.

6. Specific methods management. Finally, the Generic Tool Adapter provides mechanisms to alter the workflow of the tool. This category includes all those methods that do not fit in the previous five categories for providing functionalities that are very specific and dependent of the type of tool.

Figure 4 depicts the relationship among the e-learning system, the Generic Tool Adapter, and the third-party tool (e.g., a chatterbot). It is important to mention that the methods provided by the Generic Tool Adapter (as well as their syntax) are the same ones independently on the tool of choice.

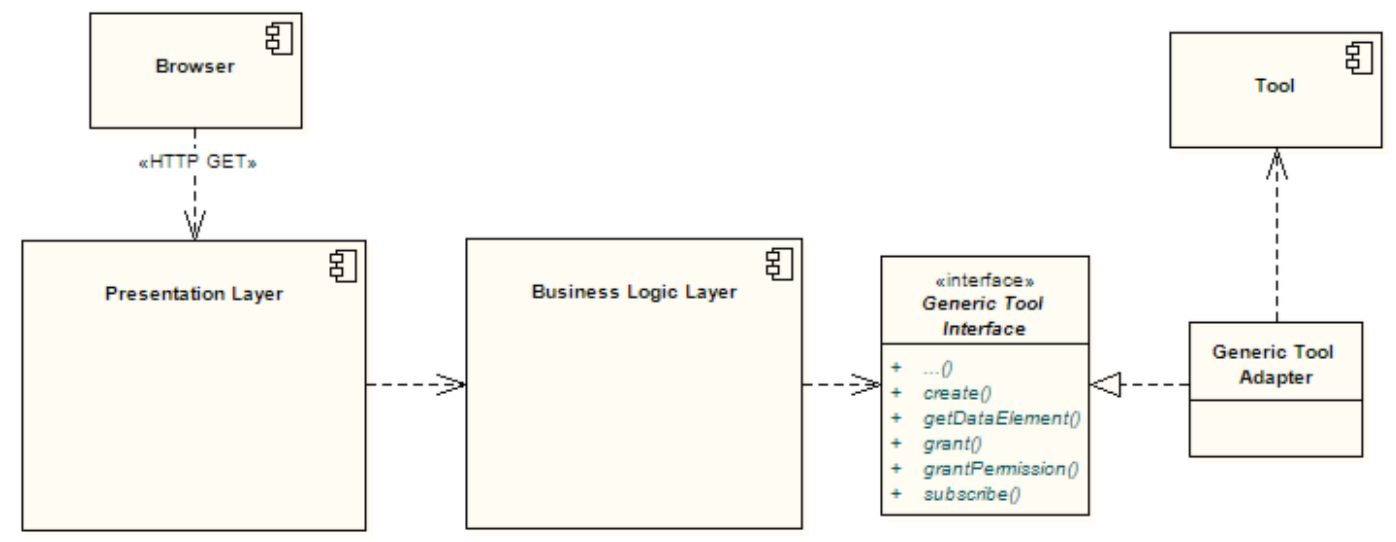

Figure 4 - Generic Tool Adapter UML component diagram

The Generic Tool Adapter features a standardized syntax to invoke its methods, i.e. it implements the Generic Tool Interface. This interface is further decomposed into six sub-interfaces, according to the six aspects of hard

Table 1 - Generic tool interface methods integration enumerated above. Table 1 summarizes some of the methods of the Generic Tool Interface, and classifies them according to the sub-interface they belong to. 


\begin{tabular}{|c|c|c|c|c|}
\hline $\begin{array}{l}\text { Sub. } \\
\text { Int. }\end{array}$ & Method & $\begin{array}{c}\text { Input } \\
\text { parameters }\end{array}$ & $\begin{array}{c}\text { Output } \\
\text { parameters }\end{array}$ & Description \\
\hline 1 & grant & $\begin{array}{l}\text { resourceURI, } \\
\text { expirationTime, } \\
\text { userName }\end{array}$ & auhtID & $\begin{array}{l}\text { Grants access to a resource given its URI, } \\
\text { the expiration time and the username of the } \\
\text { beneficiary of the authorization. Returns an } \\
\text { identifier for future references to the } \\
\text { authorization. }\end{array}$ \\
\hline 1 & revoke & authID & result & $\begin{array}{l}\text { Revokes a previous authorization given its } \\
\text { authID. Returns an error code, if any. }\end{array}$ \\
\hline 2 & createInstance & Name & instanceURI & $\begin{array}{l}\text { Creates a new instance given its name. } \\
\text { Returns its URI. }\end{array}$ \\
\hline 2 & deleteInstance & instanceURI & result & $\begin{array}{l}\text { Deletes an instance given its URI. Returns an } \\
\text { error code, if any. }\end{array}$ \\
\hline 3 & getDataElement & dataURI & data & $\begin{array}{l}\text { Requests a data element by its URI. Returns } \\
\text { its value. }\end{array}$ \\
\hline 3 & setDataElement & dataURI, data & result & $\begin{array}{l}\text { Overwrites the current value of the data } \\
\text { element given by the parameter dataUR with } \\
\text { the value contained in the parameter data. } \\
\text { Returns an error code, if any. }\end{array}$ \\
\hline 3 & getBackup & $\begin{array}{l}\text { instanceURI, } \\
\text { incremental }\end{array}$ & data & $\begin{array}{l}\text { Requests a backup copy of the data of a } \\
\text { instance given its URI. It can be a complete } \\
\text { or an incremental copy. Returns the backup } \\
\text { copy. }\end{array}$ \\
\hline 4 & grantPermission & $\begin{array}{l}\text { permission, } \\
\text { username, } \\
\text { dataURI, } \\
\text { expirationTime, } \\
\text { instanceURI }\end{array}$ & result & $\begin{array}{l}\text { Grants the given permission to a user over a } \\
\text { particular resource. If the parameter } \\
\text { dataURI is not present, it applies to all the } \\
\text { resources of the instance given by the } \\
\text { parameter instanceURI. Returns an error } \\
\text { code, if any. }\end{array}$ \\
\hline 4 & resetToDefaults & $\begin{array}{c}\text { userName, } \\
\text { dataURI, } \\
\text { instanceURI }\end{array}$ & result & $\begin{array}{l}\text { Resets the permission of the given user of } \\
\text { the given instance over the given data } \\
\text { element to their default values. }\end{array}$ \\
\hline 5 & subscribe & $\begin{array}{l}\text { event, } \\
\text { instanceURI, } \\
\text { compact }\end{array}$ & result & $\begin{array}{l}\text { Subscribe to the given event. If the } \\
\text { parameter instanceURI is present, the } \\
\text { subscription only affects to the events that } \\
\text { take place within the given instance. If the } \\
\text { parameter compact is present, similar events } \\
\text { are grouped and sent in a single message. } \\
\text { Returns an error code, if any. }\end{array}$ \\
\hline 5 & notify & $\begin{array}{c}\text { event, } \\
\text { instanceURI, } \\
\text { username }\end{array}$ & result & $\begin{array}{l}\text { Given a username and an instance URI he } \\
\text { belongs to, notifies an event to the user. } \\
\text { Returns an error code, if any. }\end{array}$ \\
\hline 6 & invoke & $\begin{array}{l}\text { methodName, } \\
\text { parameterList }\end{array}$ & data & $\begin{array}{l}\text { Invokes the given remote method with the } \\
\text { given parameters list. Returns the result in a } \\
\text { serialized format. }\end{array}$ \\
\hline
\end{tabular}

\subsection{The generic tool adapter protocol stack}

The internal architecture of the Generic Tool Adapter is based on the well-accepted approach to software design of protocol stacks. Figure 5 depicts a representation of the Generic Tool Adapter as a refinement of the TCP/IP protocol stack where the Application layer has been further divided into three sublayers, and the Generic Tool Adapter corresponds to the "Integration Manager" and "Integration Protocol" sublayers. As in the standard TCP/IP protocol stack there is a (virtual) direct communication between analogous (sub)layers, so that Integration Managers communicate with Integration Managers and Integration Protocols with Integration Protocols.

Apart from the classical Physical, Link, Network and Transport layers, three more elements require our attention:

- High-Level Entities. The LMS and the third-party tool. They represent the core of the e-learning system. They use the Integration Managers to communicate and complement each other.

- Integration Managers. A set of software components used by both High-Level Entities to allow the supervision and control of the workflow of the tool by the e-learning system. In other words, 
each Integration Manager carries out a different task to achieve hard integration.
- Integration Protocols. A set of protocols to allow the actual communication between Integration Managers.

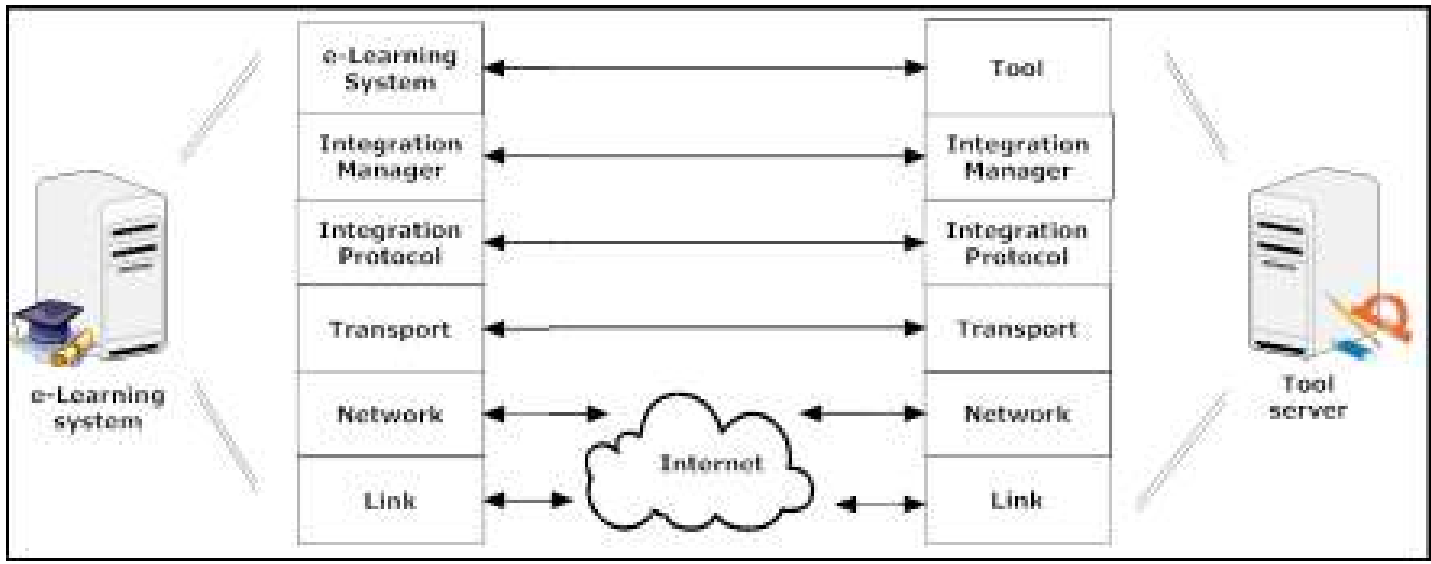

Figure 5 - Representation of the Generic Tool Adapter as a protocol stack

The Integration Managers implement the methods of the Generic Tool Interface (see Table 1) and, together with the Integration Protocols, form the Generic Tool Adapter (see Section 4.2). There are six Integration Managers and six Integration Protocols altogether. These Managers and Protocols are grouped in pairs, dealing with a specific issue of hard integration (see Section 4.1). When a method of the Integration Manager is invoked it serializes the call and forwards it to the corresponding Integration Protocol, which in turn submits it to the remote Integration Protocol. At this point, the remote Integration Protocol passes the call to the remote Integration Manager, which executes the action requested.

\section{Integrating TQ-BOT}

In order to prove the usefulness of the Generic Tool Adapter in extending the functionality of an e-learning system we decided to apply it to integrate TQ-Bot (MIKIC et al., 2009). TQ-Bot is a chatterbot based on AIML and dedicated to tutoring students, taking advantage of AI techniques and offering an appealing interface to users. This section introduces the functionalities and underlying architecture of TQ-Bot, and provides a thorough description (both static and dynamic) of the different elements of the system resulting from the combination of TQ-Bot and a generic e-learning system.

\subsection{TQ-Bot}

TQ-Bot is a virtual assistant designed for tutoring tasks, helping students in the elearning process within an e-learning system. More specifically, using TQ-Bot students are able to auto-evaluate their knowledge and skills and to ask for specific course contents. It can attract and keep students' attention because it supposes a technological innovation. Even to some degree, TQ-Bot can make the student feel more comfortable than just surfing through the learning resources and activities.

TQ-Bot is an AIML-based chatterbot, a type of conversational agent (a computer program) designed to simulate an intelligent and natural-language conversation. It processes the users' inputs and consults its knowledge base to make a response that imitates the human's one.

AIML is an XML based programming language and it is widely used in the development of software agents that communicate with their users in natural language (the programming language AIML was developed by Dr. Richard Wallace and the A.L.I.C.E.bot open source community among 1995 and 2000). AIML is a text file with a specific structure, which constitutes 
the knowledge base of the chatterbot. The "categories" are the fundamental knowledge basis, and they consist of at least two elements: the "pattern" and the "template". In general, the performance of AIML is based on a stimulusresponse model, in which the stimulus (the user's input) corresponds with the "pattern", and the response (which the chatterbot will show to the user) will be its associated "template". All these actions, about looking for the adequate pattern and showing the related template, will be carried out by a data treatment engine, of which there are many versions (Program D, Program E, etc.).

TQ-Bot has been developed as a PHP application based on Program E (KOOTSTRA, 2002), which is the PHP implementation of the AIML interpreter. TQ-Bot also uses AJAX (Asynchronous JavaScript And XML) technology, that enables to make interactive applications or RIA (Rich Internet Applications). This technique enables our bot to maintain an asynchronous communication with its server in the background, and so, it is possible to make changes on the chatterbot interface. This means a significant improvement of the interactivity.

Students interact with the bot through the BUI (Bot User Interface), which consists in a pop-up window with a text area reflecting the conversation and a text box to introduce new requests. The bot obtains input data from this BUI and searches into its knowledge source appropriate content to reply. This content is provided during the configuration of the chatterbot instance.

If the bot does not detect any input related to the content of a course, it replies to the student with an expression taken from its general knowledge base. Once the bot detects a reply from the student, where he/she has used a special keyword (related to a learning resource of the course), the bot retrieves the previously established association and processes the learning path. All needed information is found at the database tables, and TQ-Bot shows an answer consisting of:

- The resource's abstract.

- Extra information about the resource: a link to all the content of the course related to the concept that the student was asking for.

- Related information: a set of links to any type of information related to the resource that the bot has found.

- Scoring the answer: the bot offers to the student the possibility of ranking the given answer.

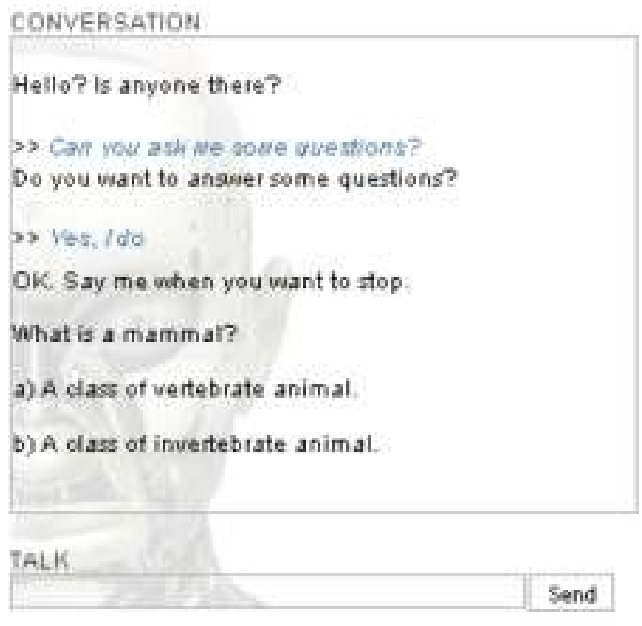

Figure 6 - Answer of the TQ-Bot

TQ-Bot also enables to auto-evaluate and monitor student progress. While a student is talking to the bot, he/she can request several activities (see Figure 6):

- To ask for a test: the bot chooses the first from all available tests that the student has not done yet.

- To ask for a personalized test: the student must choose the number of questions to be included in the test and the bot composes it.

- To ask for questions that do not belong to any test (free questions): the bot starts to ask questions and keeps on doing it until the student wants to stop.

Finally, we would like to point out that the student can ask for a clue to answer a question, and that this fact penalizes his/her final score.

\subsection{Global architecture}

In this section we describe the final architecture that allows the integration of TQ-Bot in an e-learning system. The 
architecture of TQ-Bot, the Engine, and the Generic Tool Adapter are glued together by means of the Chatterbot Binding Adapter and the Creational API. Therefore, this section is devoted to describe these two elements. The result is depicted in the UML component diagram of Figure 7.

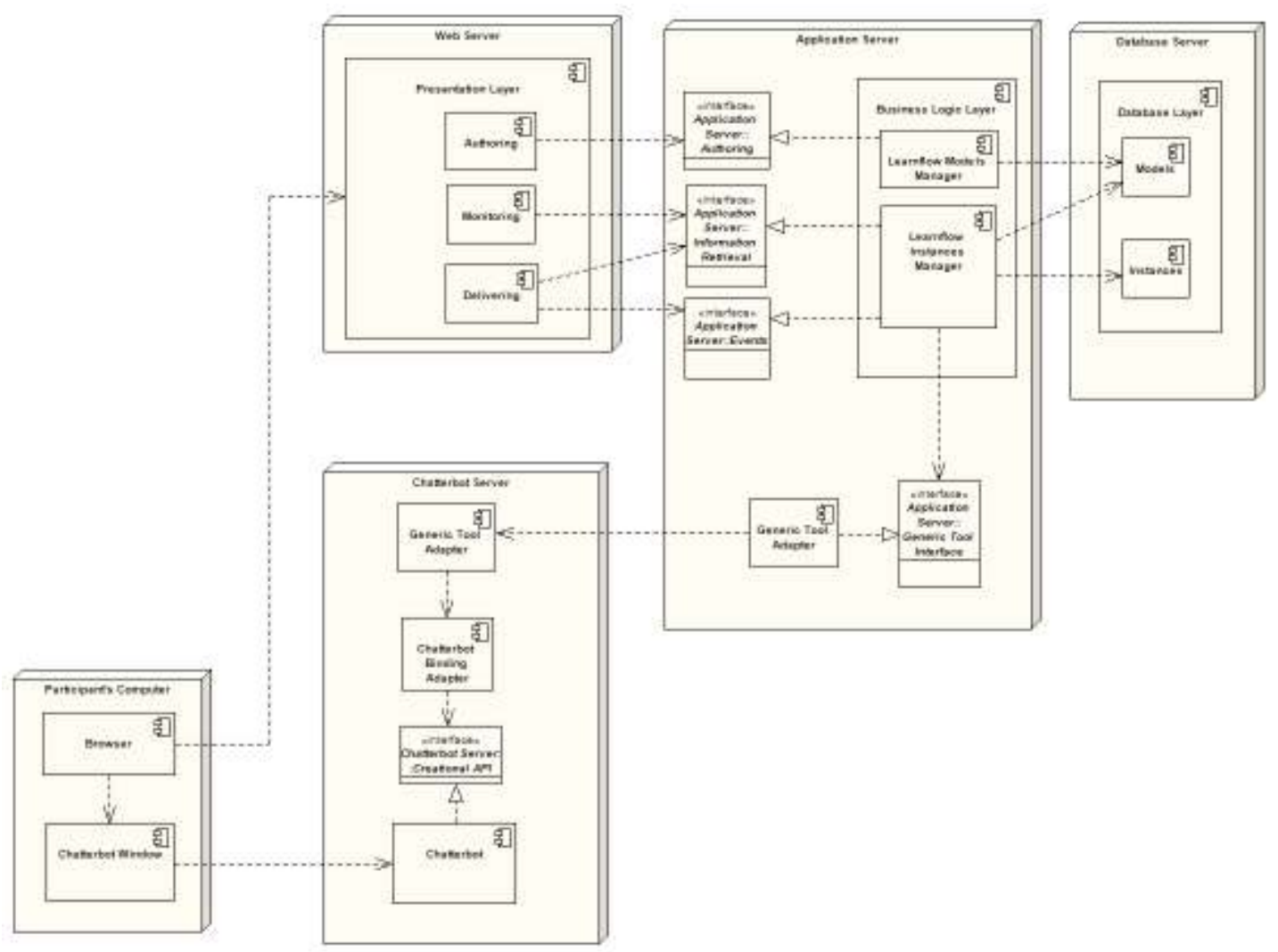

Figure 7 - UML deployment of the integration architecture

The Creational API has been posed to allow a programmatic management of the bot. Originally, the logic of TQ-Bot had been designed together with a graphical user interface that allows its configuration and management by users (namely, a teacher). This approach proved to be tiresome when the teacher has to configure a large number of instances of TQ-Bot for its students. Therefore, we defined the Creational API to enable an automated configuration of the bot by the e-learning system.

The Creational API provides the following features, in accordance with the six aspects of hard integration described in Section 4.1:

1. Authorization granting: transparent access for users to the TQ-Bot server.

2. Instances management: automated creation and deletion of instances of
TQ-Bot, and addition and removal of participants to specific instances.

3. Data transfer: methods to allow the elearning system to read and post messages in a TQ-Bot instance. An excerpt of the vocabulary used to exchange data between the e-learning system and TQ-Bot is summarized in Table 2. This vocabulary also includes terms to inform the bot about the course structure and organization.

4. Permissions assignment: methods to assign permissions to the participants of a TQ-bot instance.

5. Event subscription: methods that allow an e-learning system to subscribe to events that take place in a TQ-Bot instance. This is especially useful in educational scenarios where the elearning system must be "in touch" with 
the interaction between the user and a TQ-Bot instance.

6. Other methods: this category includes all those methods that do not fit in the previous five categories for providing functionalities that are very specific of the TQ-Bot system. We consider, for example, configuring TQ-Bot to display a message to the users of an instance (e.g. "By the way, I remind you that only 10 minutes remain to finish the test"). In addition, there are methos to inform the bot about the course structure and organization.

Table 2 - Voabulary used to configue TQ-Bot

\begin{tabular}{|c|c|}
\hline Property & Value \\
\hline Name & TQ-BOT \\
\hline Gender & Male \\
\hline Master & Fernando Mikic \\
\hline Birthday & January 1, 2007 \\
\hline Birthplace & University of Vigo \\
\hline Favouritebook & I, Robot \\
\hline Favouriteband & Smashing Punkins \\
\hline Favouritesong & Stairway to Heaven \\
\hline Favouritemovie & Matrix \\
\hline Forfun & Surfing the WWW \\
\hline Language & English \\
\hline Image & Angel.jpg \\
\hline
\end{tabular}

The Chatterbot Binding Adapter is an intermediate layer between the Creational API and the Generic Tool Interface. The reason of its existence is that, while the Generic Tool Interface has been designed for general-purpose tools (featuring generic methods such as createInstance()), the Creational API features a TQ-Bot-oriented syntax (e.g. newTQInstance()). Therefore, the purpose of the Chatterbot Binging Adapter is to perform a conversión between both syntaxes. This is in agreement with the Adapter design pattern (GAMMA et al., 1995).

The conversions between the Generic Tool Interface and the Creational API carried out by the Chatterbot Binding Adapter are actually one to one, because the latter has been designed to cover a set of common needs in learning tools. The output of the Chatterbot Binding Adapter is a request that can be appropriately processed by the Creational API. Table 3 shows the conversions that are carried out by the Chatterbot Binding Adapter.
Table 3 - Conversions carried out by the Chatterbot Binding Adapter in response to methods of Table 1

\begin{tabular}{|c|c|}
\hline Incoming request & Converted request \\
\hline grant & addParticipant \\
\hline revoke & removeParticipant \\
\hline createInstance & nwTQInstance \\
\hline deleteInstance & deleteTQInstance \\
\hline getDataElement & getTQVariable \\
\hline setDataElement & setTQVariable \\
\hline getBackup & getConversation \\
\hline Subscribe & Subscribe \\
\hline Notify & notifyToParticipant \\
\hline grantPermission & setPermission \\
\hline resetToDefaults & requestPermission \\
\hline Invoke (postMessage) & postMessage \\
\hline
\end{tabular}

\subsection{Dynamic behaviour}

In order to complement the static description of the system detailed in the previous section, here we briefly give a dynamic description of its main components. Figure 8 depicts a simplified UML sequence diagram summarizing the calls among the main entities of the architecture.

Firstly, a student using a Web browser joins the e-learning system to continue a lesson. When the user chooses a subject the browser makes a background invocation of the importCourse() JavaScript method at the LMS core. This invocation results on another method invocation by the LMS Core to the Engine API, the getES() method, requesting the current user activities.

When the LMS Core receives the reply from the Engine it checks whether or not the activities of the user involve an instance of TQ-Bot. If they do, the LMS Core makes an invocation of the method getEnvironment() of the Engine API to request the AIML files to be loaded at the chatterbot. Besides, it is possible to request additional parameters to customize the bot giving it a name, hobbies, hometown, birthday, a background image for the conversation window, structure and organization of the course (see Table 2).

At this point of the process the LMS Core knows that the course involves the use of an instance of TQ-Bot, and the configuration parameters of the instance for the current user. The next step is the creation of an instance of TQ-Bot. To that end, the LMS Core invokes the createInstance() at the Generic Tool Interface. Next, the 
configuration parameters are sent with a call to the method setDataElement() of the Generic Tool Interface. These and subsequent invocations are accordingly

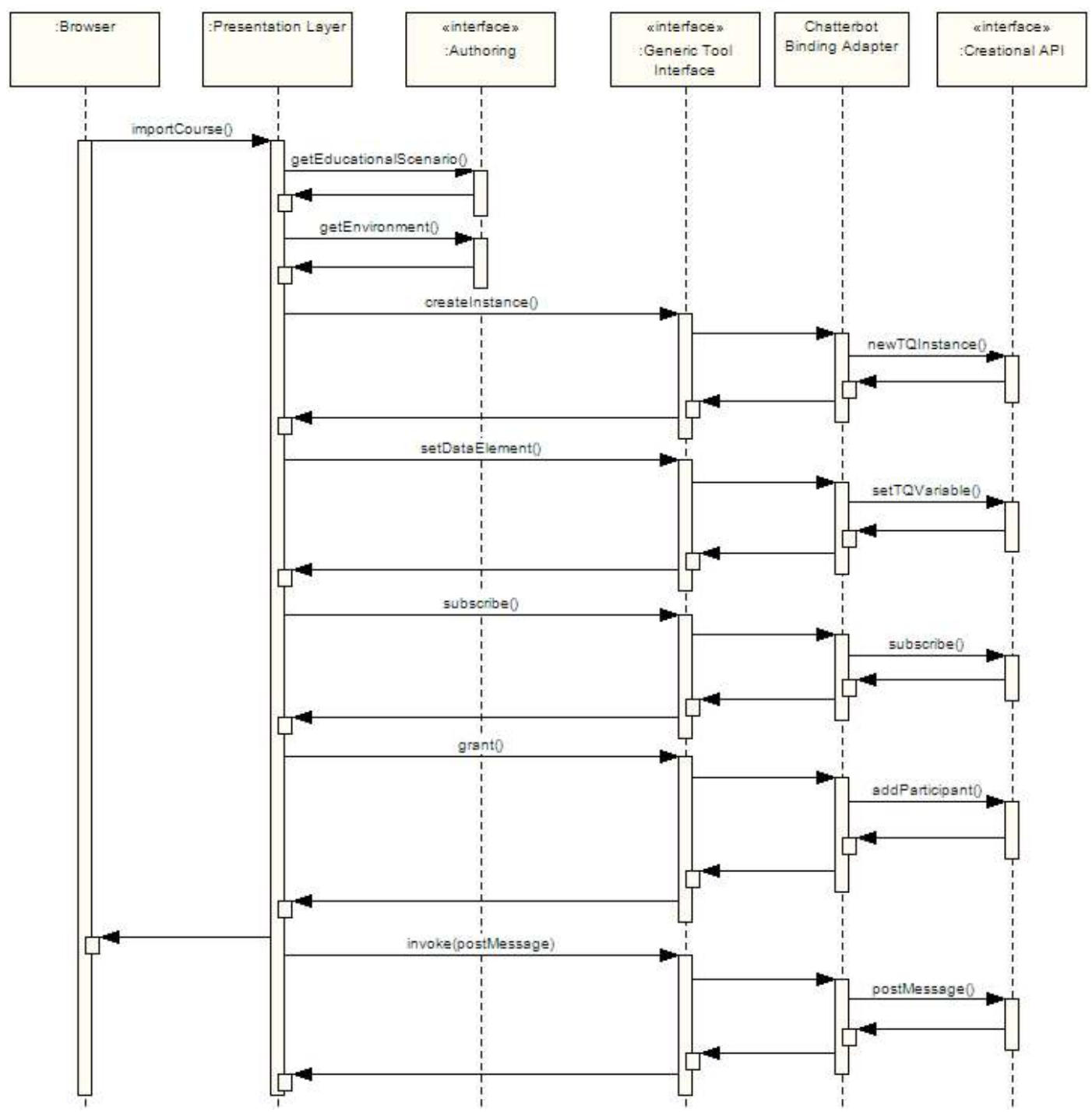

Figure 8 - UML sequence diagram of the integration architecture translated by the Chatterbot Binding Adapter in terms that can be understood by the Creational API (see Figure 8).

Figure 8 shows the actions triggered by an invocation of the postMessage() method of TQ-Bot to display a message to the user.

\section{Conclusions}

During the last years LMSs have become very popular e-learning systems. They are used by academic institutions and companies to support learning programs and educational activities. Nevertheless, there are many problems and limitations that 
remain to be solved in LMSs. A main issue is related with the isolation of learners and the lack of a tutor figure that provides companion and guidance orienting the student to the more appropriate course contents. The use of an artificial intelligence entity (an AIML-based chatterbot) can provide this functionality.

The key contribution of this piece of research is a middleware to integrate chatterbots in e-learning systems. This middleware has been developed in a generic way, not just focused on chatterbots but also on other tools that can be used in elearning: simulators, games, production tools, etc. Eventually, all these tools share some basic integration needs (managing instances, assigning permissions, etc.). Our middleware provides support to these needs following a modular approach as well as it supports specific issues on particular tools. In this paper it is shown how this middleware can be used to integrate a chatterbot in a LMS. The final integration of the TQ-Bot was achieved through the programming on a single software component: the Chatterbot Binding Adapter. Similarly, following the same approach a broad variety of tools can be integrated in the LMS. The difficulties are on the availability of a component implementing an interface with methods as the ones of the Creational API. If this component does not exist it needs to be provided.

This piece of research is in the context of recent standardization initiatives to solve the integration of third-party tools in elearning systems. These initiatives have focused in very basic problems, specifically single sing-on authentication and some degree of data-transfer. In any case, they are in an early development stage. Current e-learning systems have began to support this kind of standards very recently. The work described in this paper goes a step further involving integration issues that currently are not in the focus by the standardization community, but we are sure they will be considered in the future.

\section{Acknowledgement}

This work was partially supported by the Xunta de Galicia under INCITE (Project Number 10 PXIB 322039 PR) and by the Spanish Ministerio de Ciencia e Innovación under grant "Methodologies, Architectures and Standards for Adaptive and Accessible E-learning (Adapt2Learn)" (TIM2010-21735-C02-01)

\section{References}

ALCOM, B., VENTO, C., et al. IMS Tools Interoperability specification, IMS Specifications, 2006. Web site: [Last accessed: July 2011]. URL http://www.imsglobal.org/ti/index.html

ALICE A. I. Foundation. A.L.I.C.E. Artificial Intelligence Foundation, 1995. Web site: [Last accessed: July 2011]. URL http://alicebot.blogspot.com/

APACHE. Tomcat, 2000. Web site: [Last accessed: July 2011]. URL http://tomcat.apache.org/

APACHE. Axis, 2004. Web site: [Last accessed: July 2011]. URL http://ws.apache.org/axis/

AYALA, D., NICHOL, S. Nusoap, December 2009. Web site: [Last accessed: July 2011]. URL http://sourceforge.net/projects/nusoap

BURGUILLO, J. C., 2008. Project Galaia. Web site: [Last accessed: July 2011]. URL http://papa.det.uvigo.es/ galaia/EN/

CAEIRO, M. PoEML: A separation-of-concerns proposal to instructional design. In BOTTURI, L.; STUBBS, T. (Eds.) Handbook of Visual Languages for Instructional Design: Theories and Practices , IGI Global, 2007.

CORBETT, A. T., KOEDINGER, K. R., ANDERSON, J. R. Intelligent Tutoring Systems. Elsevier Science, p. 849-874, 1997. 
CRANE, D., MCCARTHY, P.. Comet and Reverse Ajax: The Next-Generation Ajax 2.0. Apress, Berkely, CA, USA, 2008.

DAGGER, D., O'CONNOR, A., LAWLESS, S., WALSH, E., WADE, V. P. Service-oriented e-learning platforms: From monolithic systems to flexible services. IEEE Internet Computing, v. 11, n. 3, p. 28-35, 2007.

FONTENLA, J., CAEIRO, M, LLAMAS, M., ANIDO, L.. Reverse OAuth - A solution to achieve delegated authorizations in single sign-on environments. Computers \& Security, v. 28, n. 8, p. 843-856, 2009.

GAMMA, E., HELM, R., JOHNSON, R., VLISSIDES, J. Design patterns: elements of reusable object-oriented software. Addison-Wesley Professional, 1995

HAMAMO, M. Overview of IBM Information Server - Information Integration Solutions to realize Information as a Service (IaaS), 2009. Web site: [Last accessed: July 2011]. URL http://www-

06.ibm.com/jp/provision/english/no52/article3-e.html

JISC and DEST. E-Learning Framework Web site, 2004. Web site: [Last accessed: July 2011]. URL http://www.elframework.org/

JONES, M. T. Cloud Computing with Linux, 2009. Web site: [Last accessed: July 2011]. URL http://www.ibm.com/developerworks/linux/library/l-cloud-computing/

KOOTSTRA, A. Program E, 2002. Web site: [Last accessed: July 2011]. URL http://sourceforge.net/projects/programe/

Kyng, M. Computers and Design in Context, The MIT Press, 1997.

LEONHARDT, M. D., CASTRO, D. D., DUTRA, R. L., TAROUCO, L. M. R.. Elektra: Um chatterbot para uso em ambiente educacional. Renote - Revista Novas Tecnologias na Educacao, v.1, 2003.

MIKIC, F., BURGUILLO, J., LLAMAS, M. TQ-Bot: An AIML-based tutor and evaluator bot. Journal of Universal Computer Science, vol. 15, n. 7, p. 1486-1495, 2009.

MOODLE. Moodle Web site, 2002. Web site: [Last accessed: July 2011]. URL http://moodle.org/

NEVES, A. M. M., DINIZ, I., BARROS, F. A. Natural language communication via AIML plus chatterbots. In: V Symposium on Human Factors in Computer Systems, 2002.

OKI. Open Knowledge Initiative Web site, 2001. Web site: [Last accessed: July 2011]. URL http://www.okiproject.org/

ORACLE, December 2009. Oracle. Web site: [Last accessed: July 2011]. URL http://www.oracle.com

PEREZ, R., CAEIRO, M, ANIDO, L.. Enabling Process-Based Collaboration in Moodle by Using Aspectual Services. In: Proceedings of the 2009 Ninth IEEE International Conference on Advanced Learning Technologies-Volume 00. IEEE Computer Society, p. 301-302, 2009.

PIETRO, O. D., FRONTERA, G. Tutorbot: An application aiml based for web-learning. In: USKOV, V. (Ed.). CATE. ACTA Press, p. 284-290, 2004.

SCHROEDER, C., SIMON, J., et al. IMS General Web Services specification, 2005. Web site: [Last accessed: July 2011]. URL http://www.imsglobal.org/gws/

SMYTHE, C. IMS Abstract Framework specification, 2003. Web site: [Last accessed: July 2011]. URL http://www.imsglobal.org/af/index.html

TRUMBA CORPORATION. Five benefits of software as a service, 2004. Web site: [Last accessed: July 2011]. URL http://www.trumba.com/connect/knowledgecenter/software_as_a_service.aspx

UNJHEM, E., MILLS, D., et al. IMS Common Cartridge specification, 2008. Web site: [Last accessed: July 2011]. URL http://www.imsglobal.org/cc/ 
WALSH, A. E. (Ed.). UDDI, SOAP, and WSDL: The Web Services Specification Reference Book. Prentice Hall Professional Technical Reference, 2002

WCET. EduTools portal, 2009. Web site: [Last accessed: July 2011]. URL http://www.edutools.info

WEAVER, P. Preventing e-learning failure. Training and Development, v. 56, n. 8, p. 45-50, 2002.

ZEMSKY, R., et al. Thwarted innovation: what happened to e-learning and why. A report for the Weatherstation Project of the Learning Alliance at the University of Pennsylvania, 2009. Web site: [Last accessed: July 2011]. URL http://www.irhe.upenn.edu/Docs/Jun2004/ThwartedInnovation.pdf

Recebido em maio de 2011

Aprovado para publicação em junho de 2011

Manuel Caeiro-Rodríguez

Universidade de Vigo - UVigo, Vigo - España. E-mail: manuel.caeiro@det.uvigo.es

Fernando Ariel Mikic-Fonte

Universidade de Vigo - UVigo, Vigo - España. E-mail: mikic@det.uvigo.es

Jorge Fontenla-González

Universidade de Vigo - UVigo, Vigo - España. E-mail: jfontenla@det.uvigo.es

Roberto Pérez-Rodríguez

Universidade de Vigo - UVigo, Vigo - España. E-mail: rperez@det.uvigo.es

Juan Carlos Burguillo-Rial

Universidade de Vigo - UVigo, Vigo - España. E-mail: jrial@det.uvigo.es

Martín Llamas Nistal

Universidade de Vigo - UVigo, Vigo - España. E-mail: martin.llamas@det.uvigo.es 